\title{
Precursor Gas Sensor Detection and Recognition Based On Metrology Method
}

\author{
Bo Li ${ }^{1}$, Tingting $\mathrm{Li}^{1}$ and Chuanlai Yuan ${ }^{2, *}$ \\ *Address correspondence to Chuanlai Yuan; Email: chuanlai1972@163.com \\ ${ }^{1}$ School of Information Engineering, East China Jiaotong University, \\ Nanchang, 330013, PR China \\ ${ }^{2}$ College of Electrical and Information Engineering, Hunan University of \\ Technology, Zhuzhou, 412007, PR China
}

\begin{abstract}
In the detection of drugs in the field, because the inclusion complex environment exists, the obtained signal often has a poor signal to noise ratio, and the intrinsic characteristics of the target material is often drowned signal formed in the package material in the background. According to the obtained experimental data, proposed one kind based on the principal component analysis and support vector machine algorithm of gas chromatography identification sensor signal processing and recognition; the method used for detection and identification of the air in the precursor gases combine tester self-developed, obtained very good result. This paper designed and developed a chromatographic separation and sensor based on the combination of gas detection instruments, to multi gas detection instrument. On separation characteristics using chromatography, to solve the traditional single common precursor gas detection. The use of a preprocessing based on domestication, principal component analysis for feature extraction method of all kinds of gas data. This effectively avoids the sensor substrate fluctuation and gas concentration effects on body recognition, and reduces the gas sample feature vector dimension.
\end{abstract}

Keywords: Gas detection, Chromatogram sensor, PCA, Support vector machine

\section{Introduction}

At present, there are some of the more mature technology and commodities for online detection of explosive drugs, such as X-ray transmission, neutron analysis, ion mobility spectrometry; at the same time, there are some technology is booming, such as neutron detection and analysis technology, the $X$ light cone beam 3D imaging technology [1]. Drug trafficking group interior also begins to appear highly disciplined, division of work and other trends, transit, camouflage, logistics, money laundering has detailed division of labor, the means of crime become more and more refined, subtle, especially to a small amount of times for the characteristics of the human body and possession of dangerous drugs has become an important way for drug smuggling crime group [2]. On the other hand, Dutch act to explosive events as a typical representative of the use of explosives in terrorist crime has become a new means of crime, but also increasingly rampant, the crime explosion of emerging, damage alarming. It includes not only the traditional solid explosives, such as TNT, including liquid explosives appeared in recent years [3].

Ion mobility spectrometry is a relatively mature technology, which according to different material has different principle of mobility of the ions to identify the substance to be determined [4]. First of all, the sample to be detected the formation of steam or the particles are ionized ions are formed, then, generate a weak electric field, let the ion drift, in this electric field further, recording ion through the electric field time consumption, 
then the drift time to calculate the ion mobility [5]. Although the principle of the technology is more mature, but at present, the commercial instrument and the universal degree is low. Ionization reaction and product ions were measured in different types of compounds in a sample of life differences, on the other hand, gaseous reagent and testing parameters required are also different, so in practical detection process, often require a specific recognition of objects, materials and structure of drift tube for the design and manufacture [6, 7].

Due to the continuous development of China's economy and personnel, material flow is accelerating, the number of visitors in recent years into a blowout growth trend, the dangerous goods, especially like drugs and explosives such serious harm to society and people's safety items fast, accurate detection on the scene to safeguard national stability and people's life and property safety more and the more urgent and important [8]. Therefore, in expanding the opening up policy, the spirit of not only guarantee the normal passenger transport fast travel, normal goods, and close monitoring of dangerous goods in principle, intensive and rapid flow of people and goods in real time, strictly found hidden in the dangerous goods among them, has become a major challenge for the relationship between state, society and people's vital interest is a very practical significance [9].

The work of this paper is to extend the national science and technology key project undertaken in the laboratory, the main work is design developed for rapid detection equipment common precursor chemicals, can develop a rapid detection instrument for the common precursor chemicals, can distinguish many kinds of volatile out of gas. According to the needs of the target task, to distinguish the traditional metal sensor array based on gas detection. An instrument based on the modified chromatographic separation, proposes a method to detect precursor with metal oxide semiconductor sensor based on chromatographic separation. This paper did some aspects mainly: (1) Design and development of a chromatographic separation and sensor based on the combination of gas detector, gas can be a variety of common precursor was separated and sensor chromatography based detector. (2) Use of a preprocessing based on domestication, principal component analysis for data feature a use of domestication for preprocessing based on principal component analysis method, the feature extraction of data, and the analysis of chromatography for sensor signal, and achieved good results. (3) Support vector machines were divided method for multi class based on the gas, and the use of particle swarm optimization on support vector machines were divided method for multi class based on training and get gas, gas identification model for parameters of multi class sample machine was optimized.

\section{Design of Portable Gas Sensor Based On Chromatographic Separation}

\subsection{The Principle Of Gas Chromatographic Separation Technology}

Chromatographic separation technology is also called separation technology or chromatographic separation technology for chromatography. It is an effective method for separating components in complex mixtures [10]. It is the use of different substances in the body is composed of a fixed phase and flow method. It is the use of different material has different distribution composed of fixed and mobile number in the system, with different distribution when the number two on movement of these substances with flow lines, with different distribution when the number two on movement of these substances with flow lines, so that the substance is from [11]. And repeated allocation in the two phase, so that the substance is from. Many kinds of chromatography, chromatography by many species, according to the stationary phase and separation principle of different types can be adsorption chromatography, ion exchange with as stationary phase and separation of different types of principle for adsorption chromatography [12]. 
In order to make selective sensor array signal processing, avoid the recognition of complex gas we made testing instrument adopts the method of chromatographic separation technology and sensor combination method, the technology and sensor combined, the design principle diagram was shown in Figure 1, the whole system can be divided into gas circuit and the circuit part two.

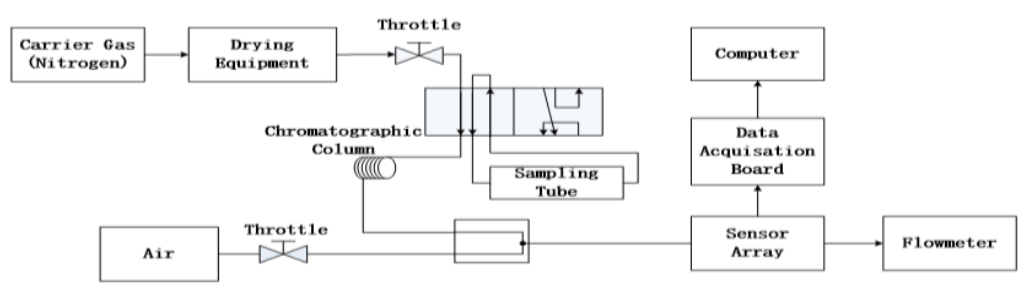

Figure 1. The Design Principle Diagram

Figure 2 is the EDXRD spectrometer we experimental structures, including $\mathrm{X}$ - ray source of white light and energy resolution of the detector with. $X$ ray white light source including $\mathrm{X}$ ray tube and the corresponding parallel light pipe, which is used to limit of the incident and scattered beams. The $\mathrm{X}$ - ray source is in the $\mathrm{W}$ target, the voltage range is $0-40 \mathrm{KV}$, the current is in the range of $0-30 \mathrm{~mA}$. The detector is using the solid high purity germanium detector cooled by liquid nitrogen; the detector has sufficient energy resolution. The detector made by Canberra, the detection area of $50 \mathrm{~mm} 2$, the crystal thickness is $5 \mathrm{~mm}$ at $5.9 \mathrm{keV}$ energy resolutions is $140 \mathrm{eV}$, and uses the Canberra spectrum master Inspector 2000 as a multichannel analyzer. X ray emitted by X ray tube, through the parallel light pipe limited beam emission angle, and then go to the two slits, exposure to the sample, the occurrence of diffraction, part of $\mathrm{X}$ ray diffraction by two after the slit into high purity germanium detector, signal acquisition by multichannel analyzer.

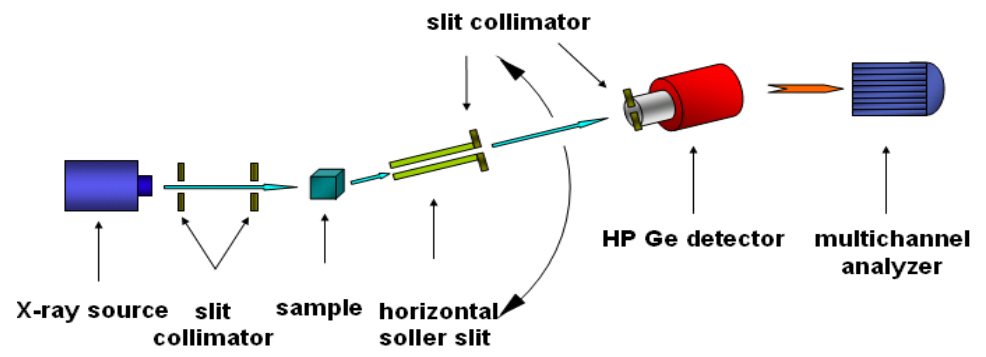

Figure 2. The EDXRD Spectrometer we Experimental Structures

\subsection{Optimization Experiments and Conditions}

When the instrument is designed and assembled, a problem of how we choose is the experimental conditions. The gas response result is affected by many factors, such as chromatography working temperature load, tail gas flow rate selection. So some condition inquiry experiment is a must. A set of gas detection instruments like is divided into two parts of gas circuit and electric circuit, relatively stable and gas is worse. So before experiment, the first thing to do is to tightness test of gas path. Leak test is a difficult but indispensable experimental part. 


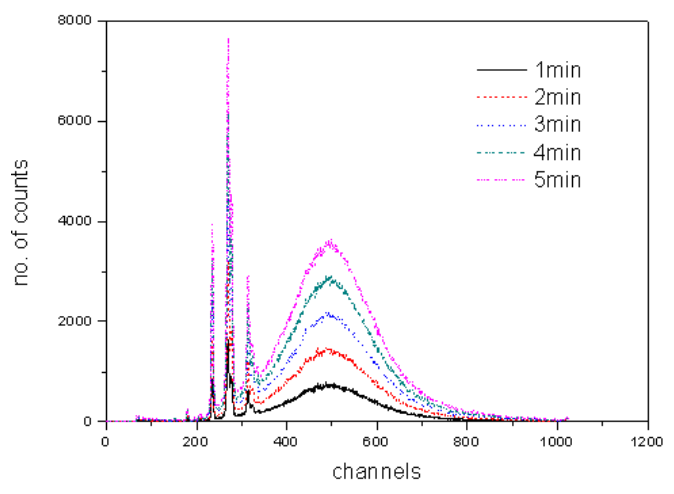

Figure 3. Chromatography Different Temperature Ether Original Signal

With the load pressure becomes larger, the response time of the sensor detects body have shorter. But with the same chromatographic operating temperature, pressure instrument identification of no gas will become shorter. In the carrier gas pressure is worse. In the carrier gas pressure is $1.4 \mathrm{MPa}$, all kinds of gas can still keep a certain distinction. In order to reduce all kinds of gas, can still maintain a certain distinction.

\subsection{Various Methods Of Precursor Gas Detection}

The chart can be seen, for a single gas sensor response in different time is due to gas in chromatography retention time is different from the. Similarly, the instrument can be of a variety of mixed test in Figure 4. Seen from the figure, the instrument components of the mixed gases also have good discrimination ability of the figure shows, the instrument components of the mixed gases also have good discrimination ability can be seen from the diagram, started in response to the at nearly the same time. The group is respectively connected with the sensor response time, start the same.
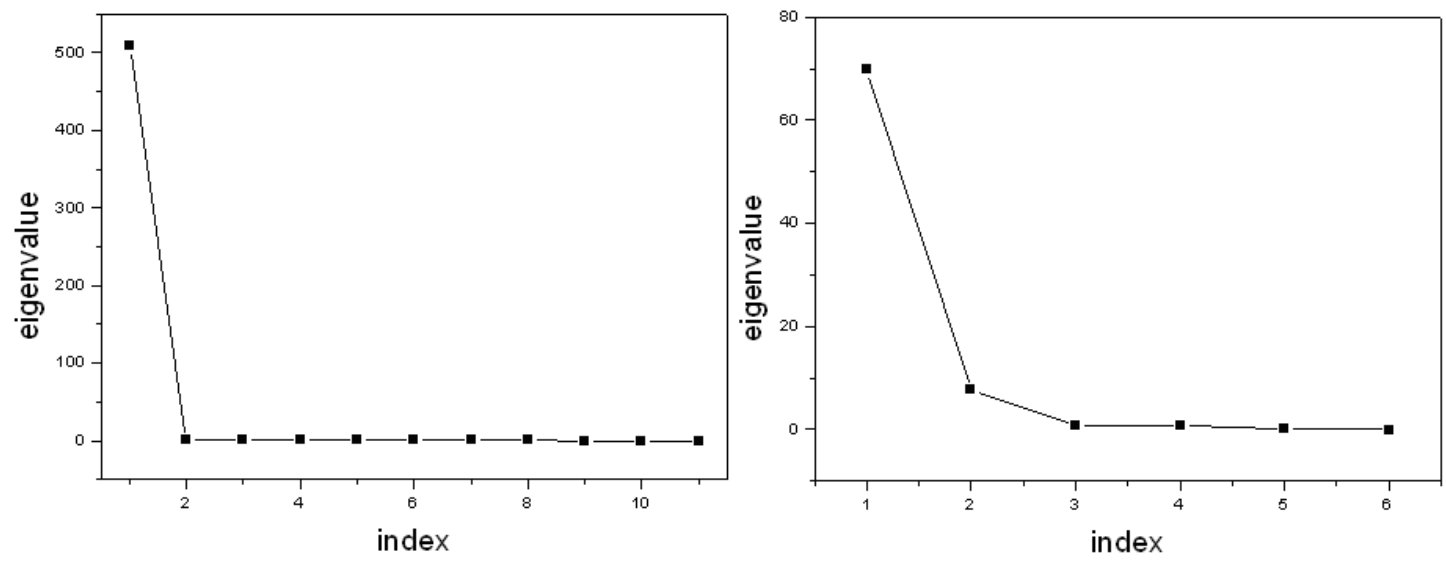

Figure 4. The Instrument can be of a Variety of Mixed Test

\section{Signal Processing Method For Gas}

\subsection{The basic idea of SVM}

SVM is the optimal class and surface development by linear separable case, for the two problem, the following to illustrate the basic idea of SVM using a two dimensional problem. $\mathrm{C} 1$ and $\mathrm{C} 2$ represent two types of data samples, each in a two-dimensional display was shown in Figure 5, a straight line in P0, P1 is a classification function. If a linear can put two of all samples, then say it is a classification function. 


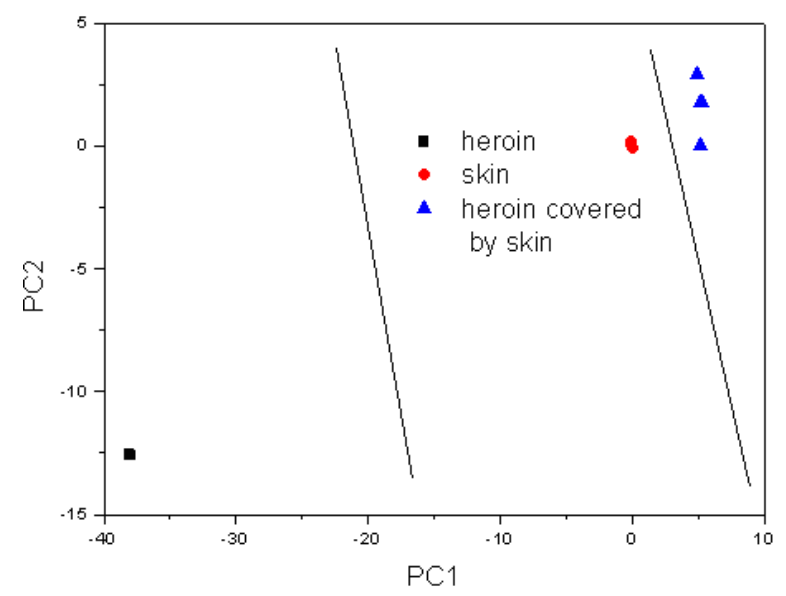

\section{Figure 5. Sketch Map of the Optimal Hyper Plane}

Now the discriminant function is normalized, so that the two types of all samples are satisfied: $|f(x)| \geq 1$, then from the surface, near the sample has $|f(x)|=1$. If all samples classified correctly to meet:

$$
y_{i}[(w * x)+b]-1 \geq 0, i=1,2, \cdots N
$$

Then the classification interval of $2\|w\|$ classification. Can find the maximum equivalent to $1 / 2\|w\|$ minimum. So the optimal classification face problems can be expressed as the following constraints:

$$
\operatorname{Min} \Phi(w)=\frac{1}{2}\|w\|^{2}
$$

The constraint conditions are:

$$
y_{i}[(w * x)+b]-1 \geq 0, i=1,2, \cdots N
$$

Define the following function:

$$
L(w, b, a)=\frac{1}{2}\|w\|^{2}-\sum_{i=1}^{N} a_{i}\left[y_{i}\left(w * x_{i}+b\right)-1\right]
$$

In the formula, $\alpha_{i} \geq 0$ Lagrange multiplier, in order to obtain the function type (4) the minimum value, respectively

$$
\left\{\begin{array}{lc}
\frac{\partial L}{\partial w}=0 \Rightarrow & w=\sum_{i=1}^{N} a_{i} y_{i} x_{i} \\
\frac{\partial L}{\partial b}=0 \Rightarrow & \sum_{i=1}^{N} a_{i} y_{i}=0 \\
\frac{\partial L}{\partial a}=0 \Rightarrow & a_{i}\left[y_{i}\left(w * x_{i}+b\right)-1\right]=0
\end{array}\right.
$$

The two function optimization problems of the existence and uniqueness of solution, if $\alpha_{\mathrm{i}}$ optimal solution, then:

$$
w^{*}=\sum_{i=1}^{N} a_{i}^{*} y_{i} x_{i}
$$

The $\alpha_{i} \neq 0$ corresponding to the support vector corresponding to the support vector. And the optimal classification face the weight coefficient vector is a linear combination of support vector machines. Classification threshold by (6) type

$$
b^{*}=-\frac{1}{2}\left(w^{*}, x_{r}+x_{s}\right)
$$




$$
a_{r}, a_{s}>0, y_{r}=-1, y_{s}=1
$$

Due to the addition of support vectors, corresponding to the non support vector $=0$, so the optimal classification function can be abbreviated as:

$$
f(x)=\operatorname{sgn}\left\{\sum_{s v} a_{i}^{*} y_{i}\left(x_{i}, x\right)+b^{*}\right\}
$$

\subsection{Kernel Function Method For Gas Recognition}

Kernel function is one of an important part of the theory of support vector machine attracted many researchers interest. The kernel function is often used to satisfy the Mercer condition are linear, polynomial and radial Sigmoid function, select different kernel can construct support vector machine function, the simple introduction to the four kinds of kernel functions

$$
\begin{array}{cc}
\text { Linear function } & K\left(x, x_{i}\right)=\left\langle x, x_{i}\right\rangle \\
\text { Polynomial function } & K\left(x, x_{i}\right)=\left[\left\langle x, x_{i}\right\rangle+1\right]^{d} \\
\text { Radial basis function } & K\left(x, x_{i}\right)=\exp \left\{-\frac{\left|x-x_{i}\right|^{2}}{\sigma^{2}}\right\} \\
\text { Sigmoid function } & K\left(x, x_{i}\right)=\tanh \left[v\left\langle x, x_{i}\right\rangle+a\right]
\end{array}
$$

\section{Results and Simulation}

\subsection{Identification of Two Species Of Gases And Gas Mixtures}

Pattern is an important link of the good performance of the computer pattern recognition system, so the method of feature extraction is very important to choose. In the actual operation, the sample data are often affected by external disturbances so that the data feature extraction more difficult. For example, in the gas detection and recognition process, the sensor from the external factors (e.g., temperature, concentration), the floating sensor signal baseline, amplitude changes with the concentration. However, our main concern is the appearance of peak time and signal, so that interference for gas qualitative identification of impact.

In order to make the sample data obtained can be more focus on the system information, first of all see data sequences are dimensionless treatment is necessary. If the sample sequence is $\mathrm{xi},(1,2, \ldots, \mathrm{N})$ there are three commonly used dimensionless method:

1) Standard method:

$$
y_{i}=x_{i} / \sum_{i=1}^{N} x_{i}\left(x_{i}>0\right)
$$

2) Normalization method:

$$
y_{i}=\left(x_{i}-\bar{x}\right) / S
$$

$$
\text { Mean value: } \bar{x}=\sum_{i=1}^{N} x_{i} / N \quad \text { variance: } S=\left(\sum_{i=1}^{N}\left(x_{i}-x\right)^{2} / N\right)^{2}
$$

3) linear normalization method:

$$
y_{i}=\left(x_{i}-\min \left\{x_{i}\right\}\right) /\left(\max _{i}\left\{x_{i}\right\}-\min _{i}\left\{x_{i}\right\}\right)
$$

\subsection{The extracted features based on PCA}

Feature extraction purpose is to effectively implement classification, the high-dimensional image vector mapping (transformation) to a low-dimensional space, get 
the best reflects the nature of the sample characteristics. So far, have been proposed many feature extraction method, which, PCA and Fisher discriminant analysis feature extraction algorithm is the most classic and widely used methods. In image recognition, PCA is the main idea: use a few features of a face image vector to represent as accurately as possible. PCA two major advantages is the elimination of the statistical correlation between the samples; dimension model samples to achieve the compression. PCA can compress high-dimensional pattern of the sample is low dimensional samples more easily handled, in other words, PCA gives a minimalist high-dimensional data representation.

NMF method is also an unsupervised learning method, NMF and PCA method is different: NMF method of weighting coefficients in the linear elements and a combination of vector-based images are all non-negative real numbers. In recent years, non-negative matrix factorization algorithm has become a hot international research. As an unsupervised method, NMF does not make full use of class information of training samples; therefore, it is likely to lose a lot of useful identification of classified information.

It can be seen from Figure 6, two-dimensional ether, mixed gas samples and three principal components, the normalized distribution is more intensive, and the mixed gas ether alcohol into powder. After normalization are densely distributed, and the mixed gas ether alcohol into powder. After normalized by the concentration, influence of different mixed voltage baseline is eliminated, so the single component dense gas well. Between the principal component and mixed gas distribution in the ether, ethanol single gas principal component results cloth.

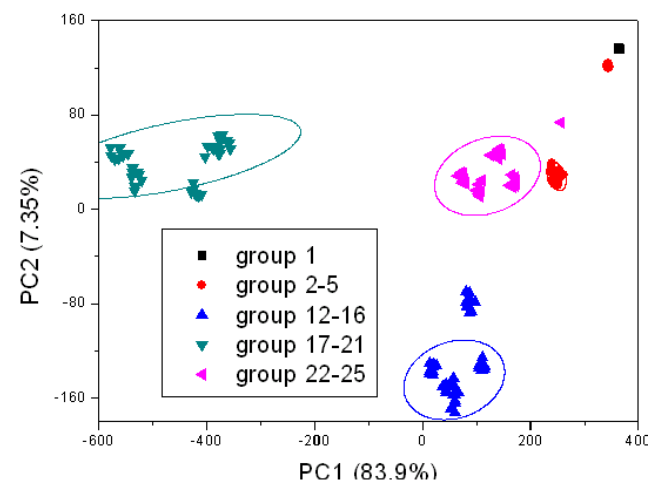

Figure 6. Two-Dimensional Ether, Mixed Gas Samples and Three Principal Components

\subsection{Study On Gas Recognition Software}

Matlab the design of graphical user interface has two methods: one is to make the $\mathrm{M}$ file programming way to establish GUI; another is the use of GUIDE graphical user interface design. The first method takes a lot of time in the position of graphical component adjustment. So we usually only used second ways, first complete control cloth and belongs to the position in the graphical component adjustment. Then select the control right click and select Object Callbacks Object Callbacks, the callback function to control to control the callback function to write operation code specific. The steps are as follows:

1) Analysis of the main function of user interface is needed, a clear design task;

2) Controls placed according to the demand, the optimization of interface;

3) The callback function to control the development of operating procedures, the realization of the corresponding function;

4) Debugging optimized program, the GUI interface is saved as a FIG resource file, automatic generation of the resource file, automatic generation of the $\mathrm{M}$ file, the end. 
The Matlab GUI interface software is already written, but this can only be installed in the machine running on PC Matlab environment, how to make the generation of executable software which can run on all PC. The main interface of gas recognition software was shown in Figure 7.

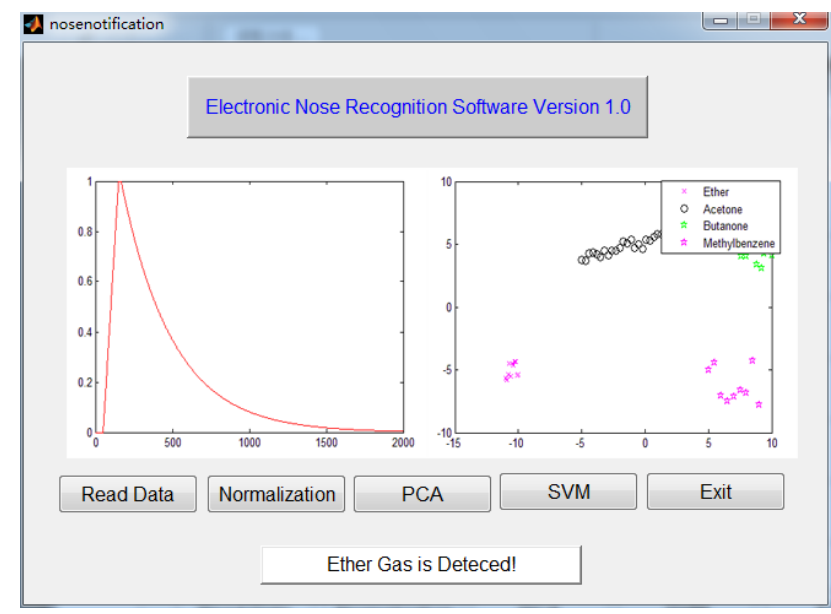

Figure 7. The Main Interface of Gas Recognition Software

\section{Conclusion}

This paper designed and developed a chromatographic separation and sensor based on the combination of gas detection instruments, to multi gas detection instrument. On separation characteristics using chromatography, to solve the traditional single common precursor gas detection. The use of a pre processing based on domestication, principal component analysis for feature extraction method of all kinds of gas data. This effectively avoids the sensor substrate voltage fluctuation and gas concentration effects on body recognition, and reduces the gas sample feature vector dimension. This paper presents a support vector machine method for multi class classified based on gas, and the use of particle swarm optimization division method for multi class gas, training and get gas recognition model. To optimize the parameters of SVM with the method, training and get the gas identification model, and achieved good results. To establish a database on the common precursor gas, using the Matlab GUI compiled a set of common gas recognition software.

\section{Acknowledgements}

This work was supported by the MOE (Ministry of Education in China) Project of Humanities and Social Sciences (12YJCZH099), the Hunan Provincial Natural Science Foundation of China (Grant No. 2015JJ5025), the Natural Science Foundation of Jiangxi Province of China (20114BAB211017) and the science and technology plan project of Jiangxi Province (20122BBE500048).

\section{References}

[1] Y. C. Su, C. C. Chang and J. L. Wang, "Construction of an automated gas chromatography/mass spectrometry system for the analysis of ambient volatile organic compounds with on-line internal standard calibration", Journal of Chromatography A, vol. 1201, no. 2, (2008), pp. 134-140.

[2] Z. Li, E. S. Demessie, A. A. Hassan and G. A. Sorial, "Transport and deposition of $\mathrm{CeO} 2$ nanoparticles in water-saturated porous media", Water Research, vol. 45, no. 15, (2011), pp. 4409-4418.

[3] N. Goldovsky and V. Goldovsky, " Correlational gas analyzer ", Measurement, vol. 33, no. 3, (2003), pp. 273-279.

[4] J. W. Leonhardt, " A new ppb-gas analyzer by means of GC-ion mobility spectrometry (GC-IMS) ", Journal of Radioanalytical and Nuclear Chemistry, vol. 257, no. 1, (2003), pp. 133-139. 
[5] J. Wang, R. Wang and D. Q. Miao, "Data enriching based on rough set theory", Journal of Environmental Sciences, vol. 29, no. 3, (2001), pp. 63-69.

[6] C. Hou, J. J. Li, D. Huo, X. G. Luo, J. 1. Dong, M. Yang and X. J. Shi, "Design of an embedded gas detector based on spectral analysis", Chinese Journal of Scientific Instrument, vol. 29, no. 4, (2008), pp. 471-475.

[7] A. Fort, M. Mugnaini, S. Rocchi, V. Vignoli, E. Comini and A. Ponzoni, " Metal-oxide nanowire sensors for CO detection: characterization and modeling ", Sensors and Actuators B: Chemical, vol. 148, no. 1, (2010), pp. 283-291.

[8] M. Breysse, B. Claudel, L. Faure, M. Guenin, J. Roberto and J. Williams, " Chemiluminescence during the catalysis of carbon monoxide oxidation on athoria surface", J. Catal, vol. 45, no. 2, (1976), pp. 137-144.

[9] X. A. Cao, Y. Tao, L. Li, Y. H. Liu, Y. Peng and J. W. Li, " An ethyl acetate sensor utilizing cataluminescence on $\mathrm{Y}_{2} \mathrm{O}_{3}$ nanoparticles", Luminescence, vol. 26, no.1, (2011), pp. 5-9.

[10] L. Tang, Y. M. Li, K. L. Xu, X. D. H. Yi, " Sensitive and selective acetone sensor based on its cataluminescence from nano- $\mathrm{La}_{2} \mathrm{O}_{3}$ surface", Sensors and Actuators B: Chemical, vol. 132, no. 1, (2008), pp. 243-249.

[11] X. A. Cao, Z. Y. Zhang, X. R. Zhang, "Sensitive a A novel gaseous acetaldehyde sensor utilizing cataluminescence on nanosized $\mathrm{BaCO}_{3}$ ", Sensors and Actuators B: Chemical, vol. 99, no. 2, (2004), pp. 30-35.

[12] Z. M. Rao, L. J. Liu, J. Y. Xie and Y. Y. Zeng, "Development of a benzene vapour sensor utilizing chemiluminescence on $\mathrm{Y}_{2} \mathrm{O}_{3}$ ", Luminescence, vol. 23, no. 3, (2008), pp. 163-168.

\section{Authors}

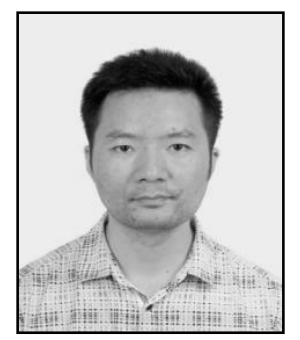

Bo $\mathbf{L i}$, received his Ph.D. in 2010 from Huazhong University of Science and Technology, China. Currently, he works as a lecturer at School of Information Engineering, East China Jiaotong University, China, and at same time doing postdoctoral research at Institute of Intelligent Machines, Chinese Academy of Sciences, China. His current research interests include pattern recognition, automatic detection and gas sensor.

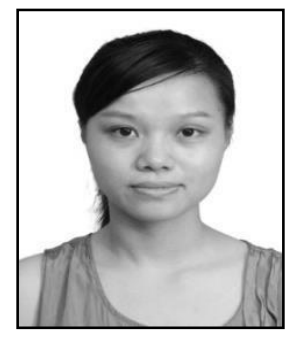

Tingting $\mathbf{L i}$, is now works as assistant at School of Information Engineering, East China Jiaotong University, China. Her current research interests include pattern recognition, automatic detection and gas sensor.

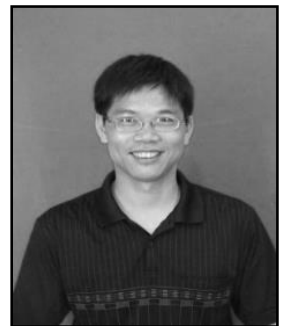

Chuanlai Yuan, received his bachelor degree in 1995 from Hunan Agricultural University, China. Currently, he works as associate professor at College of Electrical and Information Engineering, Hunan University of Technology. His current research interests include automatic detection, optimal control and design and control of industry robot. 
International Journal of Grid Distribution Computing Vol. 8, No.4, (2015) 